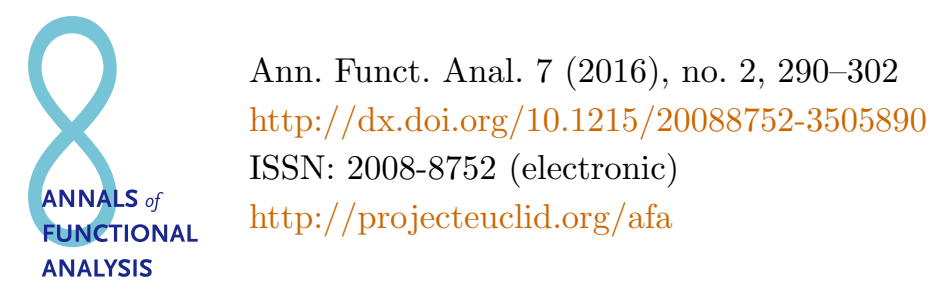

\title{
WIDE AND TIGHT SPHERICAL HULLS OF BOUNDED SETS IN BANACH SPACES
}

\author{
CHAN HE, SENLIN WU, ${ }^{*}$ and XINLING ZHANG \\ Communicated by J. Chmieliński
}

\begin{abstract}
Let $A$ be a bounded closed convex set in a Banach space. The boundaries of the wide spherical hull $\eta(A)$ and the tight spherical hull $\theta(A)$ are characterized, the existence of diametral points of these three sets are discussed, and a further relation between these three sets is clarified. Moreover, a new characterization of balls is presented.
\end{abstract}

\section{IntRoduction}

We denote by $\mathcal{H}$ the family of bounded closed sets containing at least two points in a Banach space $X$, whose origin is $o$, and by $B_{X}(x, \gamma)\left(S_{X}(x, \gamma)\right.$, resp.) the ball (sphere, resp.) centered at $x \in X$ having radius $\gamma>0$. The unit ball $B_{X}(o, 1)$ (unit sphere $S_{X}(o, 1)$, resp.) is simply denoted by $B_{X}\left(S_{X}\right.$, resp.).

For each $A \in \mathcal{H}$, we put

$$
\begin{aligned}
\gamma(A, x) & =\sup \{\|x-a\|: a \in A\}, \\
\gamma^{\prime}(A, x) & =\inf \{\|x-a\|: a \notin A\}, \quad \forall x \in X, \\
\gamma(A, B) & =\inf \{\gamma(A, x): x \in B\}, \quad \forall B \subseteq X, \\
\gamma(A) & =\gamma(A, X), \quad \gamma^{\prime}(A)=\sup \left\{\gamma^{\prime}(A, x): x \in A\right\} .
\end{aligned}
$$

Here $\gamma(A)$ is called the radius of $A$, and a point $x \in X$ satisfying $\gamma(A)=\gamma(A, x)$ will be called a center of $A$. Note that not every bounded set has a center. Suppose that $x \in A$. We say that $x$ is a diametral point of $A$ if $\gamma(A, x)=\delta(A)$,

Copyright 2016 by the Tusi Mathematical Research Group.

Received Jun. 25, 2015; Accepted Aug. 4, 2015.

${ }^{*}$ Corresponding author.

2010 Mathematics Subject Classification. Primary 46B99; Secondary 52A05, 46B20.

Keywords. complete sets, completion of sets, wide spherical hull, tight spherical hull. 


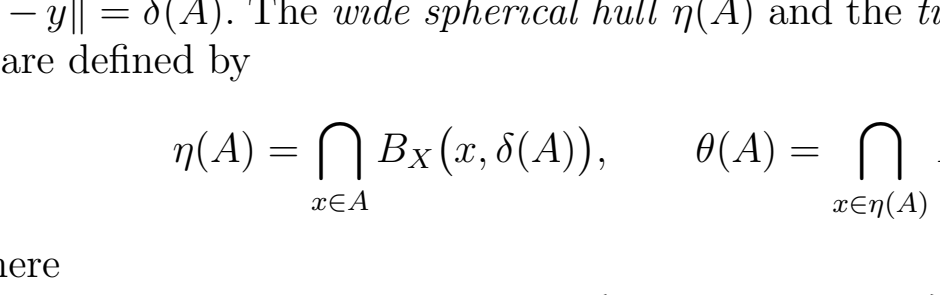

\title{
WIDE AND TIGHT SPHERICAL HULLS OF BOUNDED SETS IN BANACH SPACES
}

\author{
CHAN HE, SENLIN WU, ${ }^{*}$ and XINLING ZHANG \\ Communicated by J. Chmieliński
}

\begin{abstract}
Let $A$ be a bounded closed convex set in a Banach space. The boundaries of the wide spherical hull $\eta(A)$ and the tight spherical hull $\theta(A)$ are characterized, the existence of diametral points of these three sets are discussed, and a further relation between these three sets is clarified. Moreover, a new characterization of balls is presented.
\end{abstract}

\section{IntRoduction}

We denote by $\mathcal{H}$ the family of bounded closed sets containing at least two points in a Banach space $X$, whose origin is $o$, and by $B_{X}(x, \gamma)\left(S_{X}(x, \gamma)\right.$, resp.) the ball (sphere, resp.) centered at $x \in X$ having radius $\gamma>0$. The unit ball $B_{X}(o, 1)$ (unit sphere $S_{X}(o, 1)$, resp.) is simply denoted by $B_{X}\left(S_{X}\right.$, resp.).

For each $A \in \mathcal{H}$, we put

$$
\begin{aligned}
\gamma(A, x) & =\sup \{\|x-a\|: a \in A\}, \\
\gamma^{\prime}(A, x) & =\inf \{\|x-a\|: a \notin A\}, \quad \forall x \in X, \\
\gamma(A, B) & =\inf \{\gamma(A, x): x \in B\}, \quad \forall B \subseteq X, \\
\gamma(A) & =\gamma(A, X), \quad \gamma^{\prime}(A)=\sup \left\{\gamma^{\prime}(A, x): x \in A\right\} .
\end{aligned}
$$

Here $\gamma(A)$ is called the radius of $A$, and a point $x \in X$ satisfying $\gamma(A)=\gamma(A, x)$ will be called a center of $A$. Note that not every bounded set has a center. Suppose that $x \in A$. We say that $x$ is a diametral point of $A$ if $\gamma(A, x)=\delta(A)$,

Copyright 2016 by the Tusi Mathematical Research Group.

Received Jun. 25, 2015; Accepted Aug. 4, 2015.

${ }^{*}$ Corresponding author.

2010 Mathematics Subject Classification. Primary 46B99; Secondary 52A05, 46B20.

Keywords. complete sets, completion of sets, wide spherical hull, tight spherical hull. 


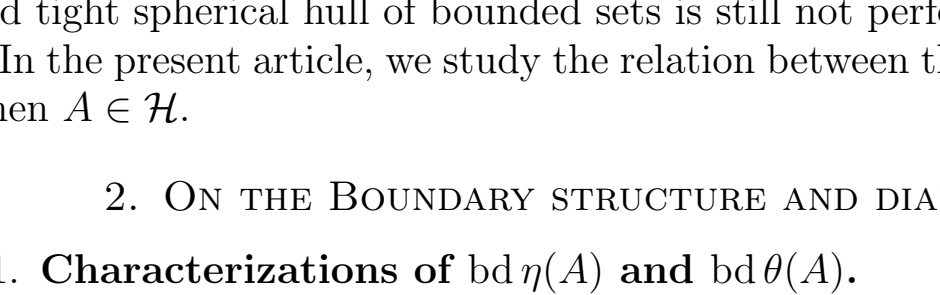

\title{
WIDE AND TIGHT SPHERICAL HULLS OF BOUNDED SETS IN BANACH SPACES
}

\author{
CHAN HE, SENLIN WU, ${ }^{*}$ and XINLING ZHANG \\ Communicated by J. Chmieliński
}

\begin{abstract}
Let $A$ be a bounded closed convex set in a Banach space. The boundaries of the wide spherical hull $\eta(A)$ and the tight spherical hull $\theta(A)$ are characterized, the existence of diametral points of these three sets are discussed, and a further relation between these three sets is clarified. Moreover, a new characterization of balls is presented.
\end{abstract}

\section{IntRoduction}

We denote by $\mathcal{H}$ the family of bounded closed sets containing at least two points in a Banach space $X$, whose origin is $o$, and by $B_{X}(x, \gamma)\left(S_{X}(x, \gamma)\right.$, resp.) the ball (sphere, resp.) centered at $x \in X$ having radius $\gamma>0$. The unit ball $B_{X}(o, 1)$ (unit sphere $S_{X}(o, 1)$, resp.) is simply denoted by $B_{X}\left(S_{X}\right.$, resp.).

For each $A \in \mathcal{H}$, we put

$$
\begin{aligned}
\gamma(A, x) & =\sup \{\|x-a\|: a \in A\}, \\
\gamma^{\prime}(A, x) & =\inf \{\|x-a\|: a \notin A\}, \quad \forall x \in X, \\
\gamma(A, B) & =\inf \{\gamma(A, x): x \in B\}, \quad \forall B \subseteq X, \\
\gamma(A) & =\gamma(A, X), \quad \gamma^{\prime}(A)=\sup \left\{\gamma^{\prime}(A, x): x \in A\right\} .
\end{aligned}
$$

Here $\gamma(A)$ is called the radius of $A$, and a point $x \in X$ satisfying $\gamma(A)=\gamma(A, x)$ will be called a center of $A$. Note that not every bounded set has a center. Suppose that $x \in A$. We say that $x$ is a diametral point of $A$ if $\gamma(A, x)=\delta(A)$,

Copyright 2016 by the Tusi Mathematical Research Group.

Received Jun. 25, 2015; Accepted Aug. 4, 2015.

${ }^{*}$ Corresponding author.

2010 Mathematics Subject Classification. Primary 46B99; Secondary 52A05, 46B20.

Keywords. complete sets, completion of sets, wide spherical hull, tight spherical hull. 


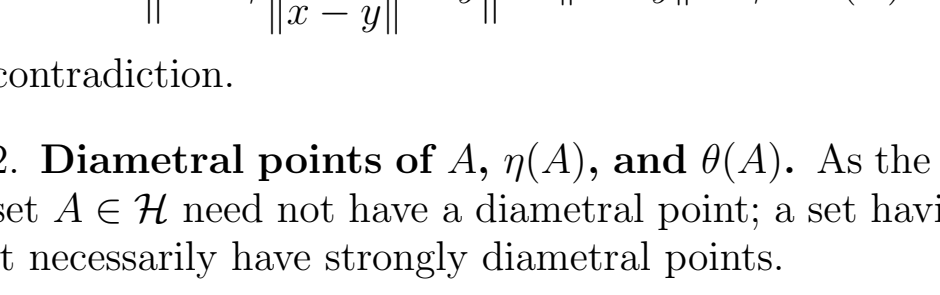

\title{
WIDE AND TIGHT SPHERICAL HULLS OF BOUNDED SETS IN BANACH SPACES
}

\author{
CHAN HE, SENLIN WU, ${ }^{*}$ and XINLING ZHANG \\ Communicated by J. Chmieliński
}

\begin{abstract}
Let $A$ be a bounded closed convex set in a Banach space. The boundaries of the wide spherical hull $\eta(A)$ and the tight spherical hull $\theta(A)$ are characterized, the existence of diametral points of these three sets are discussed, and a further relation between these three sets is clarified. Moreover, a new characterization of balls is presented.
\end{abstract}

\section{IntRoduction}

We denote by $\mathcal{H}$ the family of bounded closed sets containing at least two points in a Banach space $X$, whose origin is $o$, and by $B_{X}(x, \gamma)\left(S_{X}(x, \gamma)\right.$, resp.) the ball (sphere, resp.) centered at $x \in X$ having radius $\gamma>0$. The unit ball $B_{X}(o, 1)$ (unit sphere $S_{X}(o, 1)$, resp.) is simply denoted by $B_{X}\left(S_{X}\right.$, resp.).

For each $A \in \mathcal{H}$, we put

$$
\begin{aligned}
\gamma(A, x) & =\sup \{\|x-a\|: a \in A\}, \\
\gamma^{\prime}(A, x) & =\inf \{\|x-a\|: a \notin A\}, \quad \forall x \in X, \\
\gamma(A, B) & =\inf \{\gamma(A, x): x \in B\}, \quad \forall B \subseteq X, \\
\gamma(A) & =\gamma(A, X), \quad \gamma^{\prime}(A)=\sup \left\{\gamma^{\prime}(A, x): x \in A\right\} .
\end{aligned}
$$

Here $\gamma(A)$ is called the radius of $A$, and a point $x \in X$ satisfying $\gamma(A)=\gamma(A, x)$ will be called a center of $A$. Note that not every bounded set has a center. Suppose that $x \in A$. We say that $x$ is a diametral point of $A$ if $\gamma(A, x)=\delta(A)$,

Copyright 2016 by the Tusi Mathematical Research Group.

Received Jun. 25, 2015; Accepted Aug. 4, 2015.

${ }^{*}$ Corresponding author.

2010 Mathematics Subject Classification. Primary 46B99; Secondary 52A05, 46B20.

Keywords. complete sets, completion of sets, wide spherical hull, tight spherical hull. 


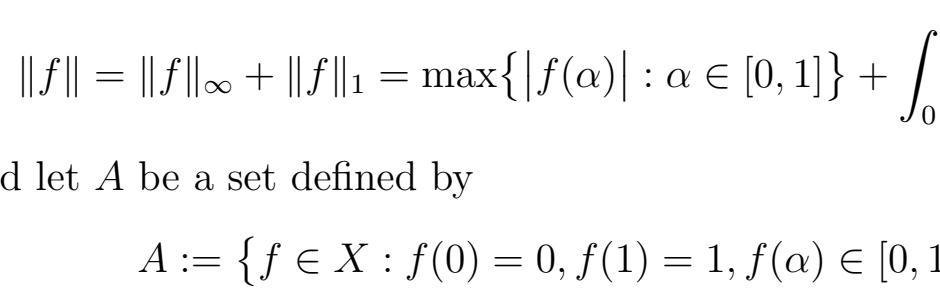

\title{
WIDE AND TIGHT SPHERICAL HULLS OF BOUNDED SETS IN BANACH SPACES
}

\author{
CHAN HE, SENLIN WU, ${ }^{*}$ and XINLING ZHANG \\ Communicated by J. Chmieliński
}

\begin{abstract}
Let $A$ be a bounded closed convex set in a Banach space. The boundaries of the wide spherical hull $\eta(A)$ and the tight spherical hull $\theta(A)$ are characterized, the existence of diametral points of these three sets are discussed, and a further relation between these three sets is clarified. Moreover, a new characterization of balls is presented.
\end{abstract}

\section{IntRoduction}

We denote by $\mathcal{H}$ the family of bounded closed sets containing at least two points in a Banach space $X$, whose origin is $o$, and by $B_{X}(x, \gamma)\left(S_{X}(x, \gamma)\right.$, resp.) the ball (sphere, resp.) centered at $x \in X$ having radius $\gamma>0$. The unit ball $B_{X}(o, 1)$ (unit sphere $S_{X}(o, 1)$, resp.) is simply denoted by $B_{X}\left(S_{X}\right.$, resp.).

For each $A \in \mathcal{H}$, we put

$$
\begin{aligned}
\gamma(A, x) & =\sup \{\|x-a\|: a \in A\}, \\
\gamma^{\prime}(A, x) & =\inf \{\|x-a\|: a \notin A\}, \quad \forall x \in X, \\
\gamma(A, B) & =\inf \{\gamma(A, x): x \in B\}, \quad \forall B \subseteq X, \\
\gamma(A) & =\gamma(A, X), \quad \gamma^{\prime}(A)=\sup \left\{\gamma^{\prime}(A, x): x \in A\right\} .
\end{aligned}
$$

Here $\gamma(A)$ is called the radius of $A$, and a point $x \in X$ satisfying $\gamma(A)=\gamma(A, x)$ will be called a center of $A$. Note that not every bounded set has a center. Suppose that $x \in A$. We say that $x$ is a diametral point of $A$ if $\gamma(A, x)=\delta(A)$,

Copyright 2016 by the Tusi Mathematical Research Group.

Received Jun. 25, 2015; Accepted Aug. 4, 2015.

${ }^{*}$ Corresponding author.

2010 Mathematics Subject Classification. Primary 46B99; Secondary 52A05, 46B20.

Keywords. complete sets, completion of sets, wide spherical hull, tight spherical hull. 


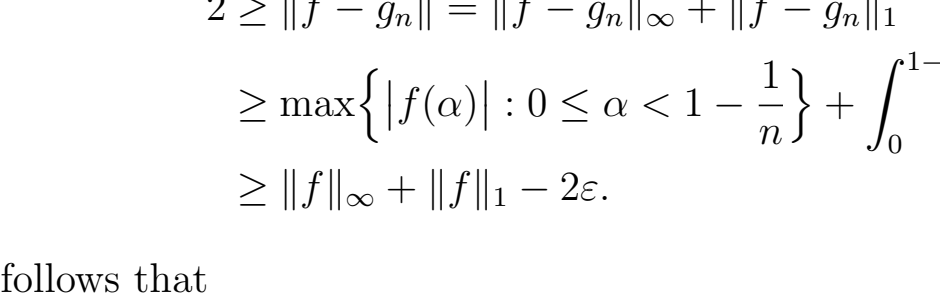

\title{
WIDE AND TIGHT SPHERICAL HULLS OF BOUNDED SETS IN BANACH SPACES
}

\author{
CHAN HE, SENLIN WU, ${ }^{*}$ and XINLING ZHANG \\ Communicated by J. Chmieliński
}

\begin{abstract}
Let $A$ be a bounded closed convex set in a Banach space. The boundaries of the wide spherical hull $\eta(A)$ and the tight spherical hull $\theta(A)$ are characterized, the existence of diametral points of these three sets are discussed, and a further relation between these three sets is clarified. Moreover, a new characterization of balls is presented.
\end{abstract}

\section{IntRoduction}

We denote by $\mathcal{H}$ the family of bounded closed sets containing at least two points in a Banach space $X$, whose origin is $o$, and by $B_{X}(x, \gamma)\left(S_{X}(x, \gamma)\right.$, resp.) the ball (sphere, resp.) centered at $x \in X$ having radius $\gamma>0$. The unit ball $B_{X}(o, 1)$ (unit sphere $S_{X}(o, 1)$, resp.) is simply denoted by $B_{X}\left(S_{X}\right.$, resp.).

For each $A \in \mathcal{H}$, we put

$$
\begin{aligned}
\gamma(A, x) & =\sup \{\|x-a\|: a \in A\}, \\
\gamma^{\prime}(A, x) & =\inf \{\|x-a\|: a \notin A\}, \quad \forall x \in X, \\
\gamma(A, B) & =\inf \{\gamma(A, x): x \in B\}, \quad \forall B \subseteq X, \\
\gamma(A) & =\gamma(A, X), \quad \gamma^{\prime}(A)=\sup \left\{\gamma^{\prime}(A, x): x \in A\right\} .
\end{aligned}
$$

Here $\gamma(A)$ is called the radius of $A$, and a point $x \in X$ satisfying $\gamma(A)=\gamma(A, x)$ will be called a center of $A$. Note that not every bounded set has a center. Suppose that $x \in A$. We say that $x$ is a diametral point of $A$ if $\gamma(A, x)=\delta(A)$,

Copyright 2016 by the Tusi Mathematical Research Group.

Received Jun. 25, 2015; Accepted Aug. 4, 2015.

${ }^{*}$ Corresponding author.

2010 Mathematics Subject Classification. Primary 46B99; Secondary 52A05, 46B20.

Keywords. complete sets, completion of sets, wide spherical hull, tight spherical hull. 


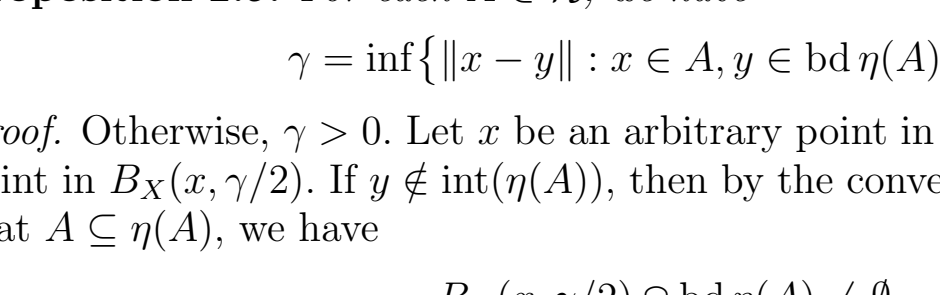

\title{
WIDE AND TIGHT SPHERICAL HULLS OF BOUNDED SETS IN BANACH SPACES
}

\author{
CHAN HE, SENLIN WU, ${ }^{*}$ and XINLING ZHANG \\ Communicated by J. Chmieliński
}

\begin{abstract}
Let $A$ be a bounded closed convex set in a Banach space. The boundaries of the wide spherical hull $\eta(A)$ and the tight spherical hull $\theta(A)$ are characterized, the existence of diametral points of these three sets are discussed, and a further relation between these three sets is clarified. Moreover, a new characterization of balls is presented.
\end{abstract}

\section{IntRoduction}

We denote by $\mathcal{H}$ the family of bounded closed sets containing at least two points in a Banach space $X$, whose origin is $o$, and by $B_{X}(x, \gamma)\left(S_{X}(x, \gamma)\right.$, resp.) the ball (sphere, resp.) centered at $x \in X$ having radius $\gamma>0$. The unit ball $B_{X}(o, 1)$ (unit sphere $S_{X}(o, 1)$, resp.) is simply denoted by $B_{X}\left(S_{X}\right.$, resp.).

For each $A \in \mathcal{H}$, we put

$$
\begin{aligned}
\gamma(A, x) & =\sup \{\|x-a\|: a \in A\}, \\
\gamma^{\prime}(A, x) & =\inf \{\|x-a\|: a \notin A\}, \quad \forall x \in X, \\
\gamma(A, B) & =\inf \{\gamma(A, x): x \in B\}, \quad \forall B \subseteq X, \\
\gamma(A) & =\gamma(A, X), \quad \gamma^{\prime}(A)=\sup \left\{\gamma^{\prime}(A, x): x \in A\right\} .
\end{aligned}
$$

Here $\gamma(A)$ is called the radius of $A$, and a point $x \in X$ satisfying $\gamma(A)=\gamma(A, x)$ will be called a center of $A$. Note that not every bounded set has a center. Suppose that $x \in A$. We say that $x$ is a diametral point of $A$ if $\gamma(A, x)=\delta(A)$,

Copyright 2016 by the Tusi Mathematical Research Group.

Received Jun. 25, 2015; Accepted Aug. 4, 2015.

${ }^{*}$ Corresponding author.

2010 Mathematics Subject Classification. Primary 46B99; Secondary 52A05, 46B20.

Keywords. complete sets, completion of sets, wide spherical hull, tight spherical hull. 


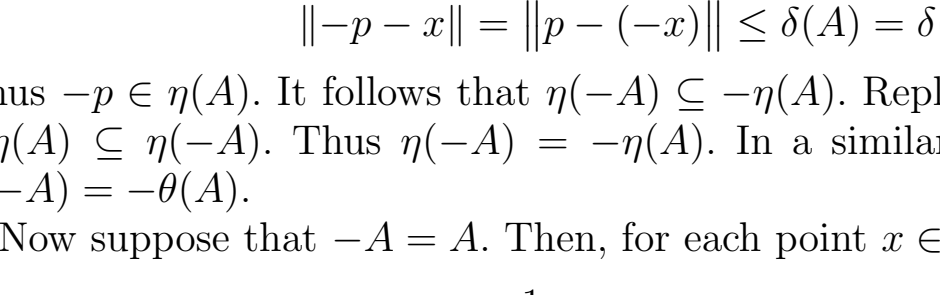

\title{
WIDE AND TIGHT SPHERICAL HULLS OF BOUNDED SETS IN BANACH SPACES
}

\author{
CHAN HE, SENLIN WU, ${ }^{*}$ and XINLING ZHANG \\ Communicated by J. Chmieliński
}

\begin{abstract}
Let $A$ be a bounded closed convex set in a Banach space. The boundaries of the wide spherical hull $\eta(A)$ and the tight spherical hull $\theta(A)$ are characterized, the existence of diametral points of these three sets are discussed, and a further relation between these three sets is clarified. Moreover, a new characterization of balls is presented.
\end{abstract}

\section{IntRoduction}

We denote by $\mathcal{H}$ the family of bounded closed sets containing at least two points in a Banach space $X$, whose origin is $o$, and by $B_{X}(x, \gamma)\left(S_{X}(x, \gamma)\right.$, resp.) the ball (sphere, resp.) centered at $x \in X$ having radius $\gamma>0$. The unit ball $B_{X}(o, 1)$ (unit sphere $S_{X}(o, 1)$, resp.) is simply denoted by $B_{X}\left(S_{X}\right.$, resp.).

For each $A \in \mathcal{H}$, we put

$$
\begin{aligned}
\gamma(A, x) & =\sup \{\|x-a\|: a \in A\}, \\
\gamma^{\prime}(A, x) & =\inf \{\|x-a\|: a \notin A\}, \quad \forall x \in X, \\
\gamma(A, B) & =\inf \{\gamma(A, x): x \in B\}, \quad \forall B \subseteq X, \\
\gamma(A) & =\gamma(A, X), \quad \gamma^{\prime}(A)=\sup \left\{\gamma^{\prime}(A, x): x \in A\right\} .
\end{aligned}
$$

Here $\gamma(A)$ is called the radius of $A$, and a point $x \in X$ satisfying $\gamma(A)=\gamma(A, x)$ will be called a center of $A$. Note that not every bounded set has a center. Suppose that $x \in A$. We say that $x$ is a diametral point of $A$ if $\gamma(A, x)=\delta(A)$,

Copyright 2016 by the Tusi Mathematical Research Group.

Received Jun. 25, 2015; Accepted Aug. 4, 2015.

${ }^{*}$ Corresponding author.

2010 Mathematics Subject Classification. Primary 46B99; Secondary 52A05, 46B20.

Keywords. complete sets, completion of sets, wide spherical hull, tight spherical hull. 


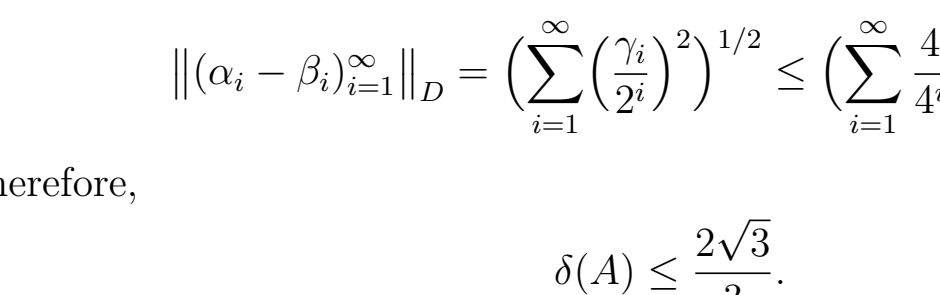

\title{
WIDE AND TIGHT SPHERICAL HULLS OF BOUNDED SETS IN BANACH SPACES
}

\author{
CHAN HE, SENLIN WU, ${ }^{*}$ and XINLING ZHANG \\ Communicated by J. Chmieliński
}

\begin{abstract}
Let $A$ be a bounded closed convex set in a Banach space. The boundaries of the wide spherical hull $\eta(A)$ and the tight spherical hull $\theta(A)$ are characterized, the existence of diametral points of these three sets are discussed, and a further relation between these three sets is clarified. Moreover, a new characterization of balls is presented.
\end{abstract}

\section{IntRoduction}

We denote by $\mathcal{H}$ the family of bounded closed sets containing at least two points in a Banach space $X$, whose origin is $o$, and by $B_{X}(x, \gamma)\left(S_{X}(x, \gamma)\right.$, resp.) the ball (sphere, resp.) centered at $x \in X$ having radius $\gamma>0$. The unit ball $B_{X}(o, 1)$ (unit sphere $S_{X}(o, 1)$, resp.) is simply denoted by $B_{X}\left(S_{X}\right.$, resp.).

For each $A \in \mathcal{H}$, we put

$$
\begin{aligned}
\gamma(A, x) & =\sup \{\|x-a\|: a \in A\}, \\
\gamma^{\prime}(A, x) & =\inf \{\|x-a\|: a \notin A\}, \quad \forall x \in X, \\
\gamma(A, B) & =\inf \{\gamma(A, x): x \in B\}, \quad \forall B \subseteq X, \\
\gamma(A) & =\gamma(A, X), \quad \gamma^{\prime}(A)=\sup \left\{\gamma^{\prime}(A, x): x \in A\right\} .
\end{aligned}
$$

Here $\gamma(A)$ is called the radius of $A$, and a point $x \in X$ satisfying $\gamma(A)=\gamma(A, x)$ will be called a center of $A$. Note that not every bounded set has a center. Suppose that $x \in A$. We say that $x$ is a diametral point of $A$ if $\gamma(A, x)=\delta(A)$,

Copyright 2016 by the Tusi Mathematical Research Group.

Received Jun. 25, 2015; Accepted Aug. 4, 2015.

${ }^{*}$ Corresponding author.

2010 Mathematics Subject Classification. Primary 46B99; Secondary 52A05, 46B20.

Keywords. complete sets, completion of sets, wide spherical hull, tight spherical hull. 


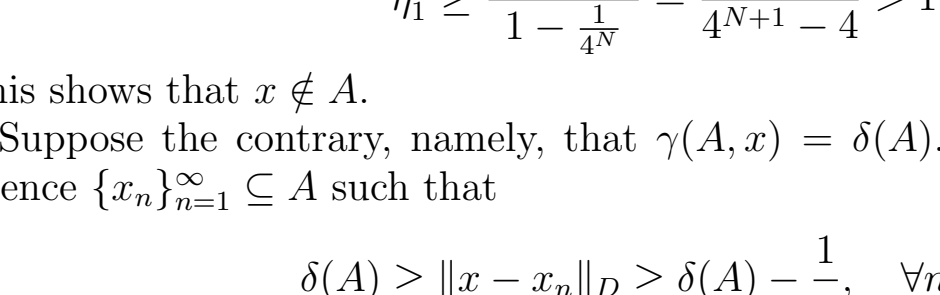

\title{
WIDE AND TIGHT SPHERICAL HULLS OF BOUNDED SETS IN BANACH SPACES
}

\author{
CHAN HE, SENLIN WU, ${ }^{*}$ and XINLING ZHANG \\ Communicated by J. Chmieliński
}

\begin{abstract}
Let $A$ be a bounded closed convex set in a Banach space. The boundaries of the wide spherical hull $\eta(A)$ and the tight spherical hull $\theta(A)$ are characterized, the existence of diametral points of these three sets are discussed, and a further relation between these three sets is clarified. Moreover, a new characterization of balls is presented.
\end{abstract}

\section{IntRoduction}

We denote by $\mathcal{H}$ the family of bounded closed sets containing at least two points in a Banach space $X$, whose origin is $o$, and by $B_{X}(x, \gamma)\left(S_{X}(x, \gamma)\right.$, resp.) the ball (sphere, resp.) centered at $x \in X$ having radius $\gamma>0$. The unit ball $B_{X}(o, 1)$ (unit sphere $S_{X}(o, 1)$, resp.) is simply denoted by $B_{X}\left(S_{X}\right.$, resp.).

For each $A \in \mathcal{H}$, we put

$$
\begin{aligned}
\gamma(A, x) & =\sup \{\|x-a\|: a \in A\}, \\
\gamma^{\prime}(A, x) & =\inf \{\|x-a\|: a \notin A\}, \quad \forall x \in X, \\
\gamma(A, B) & =\inf \{\gamma(A, x): x \in B\}, \quad \forall B \subseteq X, \\
\gamma(A) & =\gamma(A, X), \quad \gamma^{\prime}(A)=\sup \left\{\gamma^{\prime}(A, x): x \in A\right\} .
\end{aligned}
$$

Here $\gamma(A)$ is called the radius of $A$, and a point $x \in X$ satisfying $\gamma(A)=\gamma(A, x)$ will be called a center of $A$. Note that not every bounded set has a center. Suppose that $x \in A$. We say that $x$ is a diametral point of $A$ if $\gamma(A, x)=\delta(A)$,

Copyright 2016 by the Tusi Mathematical Research Group.

Received Jun. 25, 2015; Accepted Aug. 4, 2015.

${ }^{*}$ Corresponding author.

2010 Mathematics Subject Classification. Primary 46B99; Secondary 52A05, 46B20.

Keywords. complete sets, completion of sets, wide spherical hull, tight spherical hull. 


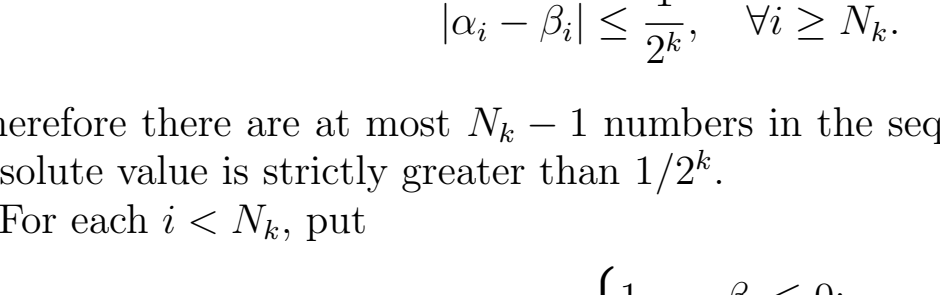

\title{
WIDE AND TIGHT SPHERICAL HULLS OF BOUNDED SETS IN BANACH SPACES
}

\author{
CHAN HE, SENLIN WU, ${ }^{*}$ and XINLING ZHANG \\ Communicated by J. Chmieliński
}

\begin{abstract}
Let $A$ be a bounded closed convex set in a Banach space. The boundaries of the wide spherical hull $\eta(A)$ and the tight spherical hull $\theta(A)$ are characterized, the existence of diametral points of these three sets are discussed, and a further relation between these three sets is clarified. Moreover, a new characterization of balls is presented.
\end{abstract}

\section{IntRoduction}

We denote by $\mathcal{H}$ the family of bounded closed sets containing at least two points in a Banach space $X$, whose origin is $o$, and by $B_{X}(x, \gamma)\left(S_{X}(x, \gamma)\right.$, resp.) the ball (sphere, resp.) centered at $x \in X$ having radius $\gamma>0$. The unit ball $B_{X}(o, 1)$ (unit sphere $S_{X}(o, 1)$, resp.) is simply denoted by $B_{X}\left(S_{X}\right.$, resp.).

For each $A \in \mathcal{H}$, we put

$$
\begin{aligned}
\gamma(A, x) & =\sup \{\|x-a\|: a \in A\}, \\
\gamma^{\prime}(A, x) & =\inf \{\|x-a\|: a \notin A\}, \quad \forall x \in X, \\
\gamma(A, B) & =\inf \{\gamma(A, x): x \in B\}, \quad \forall B \subseteq X, \\
\gamma(A) & =\gamma(A, X), \quad \gamma^{\prime}(A)=\sup \left\{\gamma^{\prime}(A, x): x \in A\right\} .
\end{aligned}
$$

Here $\gamma(A)$ is called the radius of $A$, and a point $x \in X$ satisfying $\gamma(A)=\gamma(A, x)$ will be called a center of $A$. Note that not every bounded set has a center. Suppose that $x \in A$. We say that $x$ is a diametral point of $A$ if $\gamma(A, x)=\delta(A)$,

Copyright 2016 by the Tusi Mathematical Research Group.

Received Jun. 25, 2015; Accepted Aug. 4, 2015.

${ }^{*}$ Corresponding author.

2010 Mathematics Subject Classification. Primary 46B99; Secondary 52A05, 46B20.

Keywords. complete sets, completion of sets, wide spherical hull, tight spherical hull. 


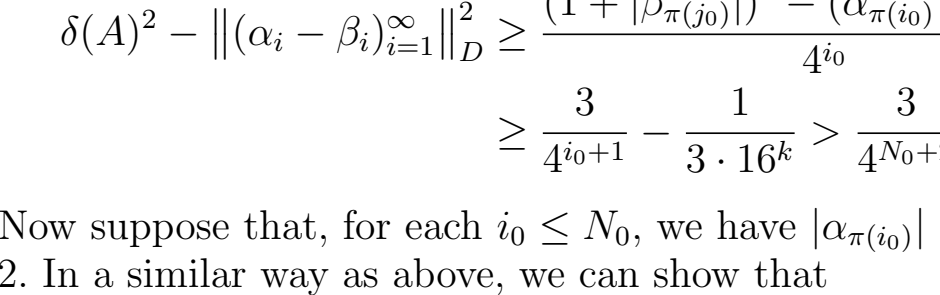

\title{
WIDE AND TIGHT SPHERICAL HULLS OF BOUNDED SETS IN BANACH SPACES
}

\author{
CHAN HE, SENLIN WU, ${ }^{*}$ and XINLING ZHANG \\ Communicated by J. Chmieliński
}

\begin{abstract}
Let $A$ be a bounded closed convex set in a Banach space. The boundaries of the wide spherical hull $\eta(A)$ and the tight spherical hull $\theta(A)$ are characterized, the existence of diametral points of these three sets are discussed, and a further relation between these three sets is clarified. Moreover, a new characterization of balls is presented.
\end{abstract}

\section{IntRoduction}

We denote by $\mathcal{H}$ the family of bounded closed sets containing at least two points in a Banach space $X$, whose origin is $o$, and by $B_{X}(x, \gamma)\left(S_{X}(x, \gamma)\right.$, resp.) the ball (sphere, resp.) centered at $x \in X$ having radius $\gamma>0$. The unit ball $B_{X}(o, 1)$ (unit sphere $S_{X}(o, 1)$, resp.) is simply denoted by $B_{X}\left(S_{X}\right.$, resp.).

For each $A \in \mathcal{H}$, we put

$$
\begin{aligned}
\gamma(A, x) & =\sup \{\|x-a\|: a \in A\}, \\
\gamma^{\prime}(A, x) & =\inf \{\|x-a\|: a \notin A\}, \quad \forall x \in X, \\
\gamma(A, B) & =\inf \{\gamma(A, x): x \in B\}, \quad \forall B \subseteq X, \\
\gamma(A) & =\gamma(A, X), \quad \gamma^{\prime}(A)=\sup \left\{\gamma^{\prime}(A, x): x \in A\right\} .
\end{aligned}
$$

Here $\gamma(A)$ is called the radius of $A$, and a point $x \in X$ satisfying $\gamma(A)=\gamma(A, x)$ will be called a center of $A$. Note that not every bounded set has a center. Suppose that $x \in A$. We say that $x$ is a diametral point of $A$ if $\gamma(A, x)=\delta(A)$,

Copyright 2016 by the Tusi Mathematical Research Group.

Received Jun. 25, 2015; Accepted Aug. 4, 2015.

${ }^{*}$ Corresponding author.

2010 Mathematics Subject Classification. Primary 46B99; Secondary 52A05, 46B20.

Keywords. complete sets, completion of sets, wide spherical hull, tight spherical hull. 


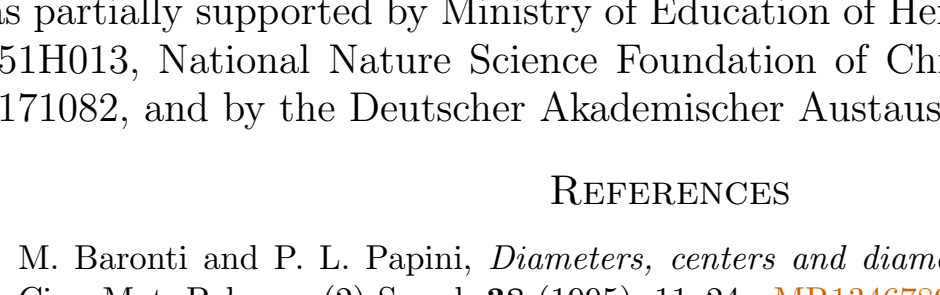

\title{
WIDE AND TIGHT SPHERICAL HULLS OF BOUNDED SETS IN BANACH SPACES
}

\author{
CHAN HE, SENLIN WU, ${ }^{*}$ and XINLING ZHANG \\ Communicated by J. Chmieliński
}

\begin{abstract}
Let $A$ be a bounded closed convex set in a Banach space. The boundaries of the wide spherical hull $\eta(A)$ and the tight spherical hull $\theta(A)$ are characterized, the existence of diametral points of these three sets are discussed, and a further relation between these three sets is clarified. Moreover, a new characterization of balls is presented.
\end{abstract}

\section{IntRoduction}

We denote by $\mathcal{H}$ the family of bounded closed sets containing at least two points in a Banach space $X$, whose origin is $o$, and by $B_{X}(x, \gamma)\left(S_{X}(x, \gamma)\right.$, resp.) the ball (sphere, resp.) centered at $x \in X$ having radius $\gamma>0$. The unit ball $B_{X}(o, 1)$ (unit sphere $S_{X}(o, 1)$, resp.) is simply denoted by $B_{X}\left(S_{X}\right.$, resp.).

For each $A \in \mathcal{H}$, we put

$$
\begin{aligned}
\gamma(A, x) & =\sup \{\|x-a\|: a \in A\}, \\
\gamma^{\prime}(A, x) & =\inf \{\|x-a\|: a \notin A\}, \quad \forall x \in X, \\
\gamma(A, B) & =\inf \{\gamma(A, x): x \in B\}, \quad \forall B \subseteq X, \\
\gamma(A) & =\gamma(A, X), \quad \gamma^{\prime}(A)=\sup \left\{\gamma^{\prime}(A, x): x \in A\right\} .
\end{aligned}
$$

Here $\gamma(A)$ is called the radius of $A$, and a point $x \in X$ satisfying $\gamma(A)=\gamma(A, x)$ will be called a center of $A$. Note that not every bounded set has a center. Suppose that $x \in A$. We say that $x$ is a diametral point of $A$ if $\gamma(A, x)=\delta(A)$,

Copyright 2016 by the Tusi Mathematical Research Group.

Received Jun. 25, 2015; Accepted Aug. 4, 2015.

${ }^{*}$ Corresponding author.

2010 Mathematics Subject Classification. Primary 46B99; Secondary 52A05, 46B20.

Keywords. complete sets, completion of sets, wide spherical hull, tight spherical hull. 